\title{
THEORY OF MEASURE AND INVARIANT INTEGRALS $\dagger$
}

\author{
BY \\ EBERHARD HOPF $\ddagger$
}

1. Introduction. Let $M$ be an analytic manifold of any number of dimensions, and let the volume measure on $M$ be denoted generally by $m$. Let $T$ be an analytic one-to-one transformation of $M$ into itself. Such a transformation may have a positive invariant integral,

$$
m^{*}(a)=\int_{a} f(P) d m
$$

where $f(P)>0$ for almost all points $P$ of $M$. The invariance property means that

$$
m^{*}\left(a_{1}\right)=m^{*}(a)
$$

holds for any measurable subset $a$ of $M, a_{1}$ being the image of $a$ under $T$. Such transformations are known to play an important rôle in dynamics. The motions of a dynamical system, considered in the manifold of states of motion, are equivalent to a one-parameter group of one-to-one transformations. In the case of a conservative system these transformations always possess a positive invariant integral; for instance in the case of a Hamiltonian system the phase volume itself is invariant.

The integral $m^{*}(a)$ may be regarded as another measure on $M$; thus a transformation of that kind is measure-preserving for a suitably chosen measure. The following paper deals with such transformations, for which the invariant measure $m^{*}(M)$ of the whole manifold $M$ is finite, and is devoted to the characterization of these transformations by their intrinsic properties.

Necessary conditions for the existence of a finite invariant measure can be easily derived. For instance no point set $a$ of positive measure can be transformed into a "proper" part of itself, i.e.

$$
a_{1} \subset a, \quad m\left(a_{1}\right)<m(a),
$$

for this would imply $m^{*}\left(a_{1}\right)<m^{*}(a)$ in contradiction to the invariance. This intrinsic property of those transformations plays an important rôle in Poincaré's and Birkhoff's work on the motions of dynamical systems. However, this is not the only intrinsic property of those transformations. It is equally

† Presented to the Society, September 11, 1931; received by the editors September 11, 1931.

$\ddagger$ International Research Fellow. 
easy to give a still stronger necessary condition for the existence of a finite invariant $m^{*}$ by introducing the concept of the "image by division."

Definition. Two measurable point sets $A$ and $A^{\prime}$ are images by division of each other, if it is possible to subdivide $A$ as well as $A^{\prime}$ into finitely or denumerably many measurable parts,

$$
A=a^{1}+a^{2}+a^{3}+\cdots, A^{\prime}=a^{(1)}+a^{(2)}+a^{(3)}+\cdots,
$$

in such a way that $a^{(\nu)}$ is an image of $a^{\nu}$ under a suitable power of $T$.

If $T$ possesses a finite invariant $m^{*}$, obviously every image by division $M^{\prime}$ of $M$ must coincide with $M$ in the sense of the theory of measure, i.e., $m(M$ $\left.-M^{\prime}\right)=0$. We have, indeed, $M=\sum a^{\nu}, M^{\prime}=\sum a^{(v)}$,

$$
m^{*}\left(M^{\prime}\right)=\sum m^{*}\left(a^{(\nu)}\right)=\sum m^{*}\left(a^{\nu}\right)=m(M),
$$

i.e., $m^{*}\left(M-M^{\prime}\right)=0$, thus yielding $m\left(M-M^{\prime}\right)=0$.

The main purpose of this paper is to show that the latter necessary condition for the existence of a finite invariant $m^{*}$ is also sufficient:

A positive invariant integral $m^{*}(a), m^{*}(M)$ being finite, can always be found, if $m\left(M-M^{\prime}\right)=0$ holds for every image by division $M^{\prime}$ of $M$.

Naturally this characterization is not fit for immediate applications, but nevertheless it throws a certain light on the intrinsic nature of those transformations. It characterizes them by an intrinsic incompressibility-property. It may be remarked that the invariant measure can be constructed by an explicit process, by introducing the concept of the "compressibility measure," of a point set with respect to another set, in generalization of a process introduced by G. D. Birkhoff and P. Smith. $\dagger$

Since we adopt the theory of Lebesgue measure as a general basis of our considerations, $\ddagger$ the assumptions of analyticity of manifold and transformation are inessential and may be replaced by much more general assumptionsई.

2. The compressibility measure. Preliminary theorems on invariant measures. Let $M$ be an abstract point set. A measure $m$ in the sense of Lebesgue may be defined on $M$ and on certain subsets of $M$ called measurable sets. Only measurable sets will be considered in this paper. These sets are supposed to satisfy the following well known conditions:

(I) The sum of finitely or denumerably many measurable sets is measurable.

$\dagger$ G. D. Birkhoff and P. Smith, Structure analysis of surface transformations, Journal de Mathematiques pures et appliquées, (9), vol. 7 (1928), p. 345.

$\ddagger$ This is a natural basis in connection with dynamics. Invariant measures occurring in this field are always absolutely additive measures. Under the weaker condition of ordinary additivity an invariant measure exists without any condition; see I. von Neumann, Zur allgemeinen Theorie des Masses, Fundamenta Mathematicae, vol. 12 (1928), p. 73.

$\S$ I wish to express my gratitude to Professor Birkhoff for suggesting to me work in this field. 
(II) If $a$ and $b$ are measurable and $a c b$, then $b-a$ is measurable.

As a well known consequence of (I) and (II) the set $a b$ of all points common to $a$ and $b$ is measurable, for it is $a b=b-((a+b)-a)$. The measure $m$ is supposed to have the following properties:

(i) $m \geqq 0$.

(ii) $m$ is absolutely additive,

$$
m(a+b+c+\cdots)=m(a)+m(b)+m(c)+\cdots
$$

for finitely or denumerably many sets $a, b, c, \cdots$ excluding each other.

(iii) Each set $a$ of positive measure contains a set $b$ with

$$
0<m(b)<m(a) .
$$

(iv) $m(M)$ is positive and finite.

The condition (iii) merely excludes triviality. The following considerations are based on this measure as a standard measure.

For our purposes we have to take into account different measures $m^{*}$ being comparable with $m$ in the following sense:

1. $m^{*}$ is defined for the same sets as $m$, i.e., for all measurable sets introduced above.

2. $m^{*} \geqq 0$.

3. $m^{*}$ is absolutely additive.

4. The relations $m=0$ and $m^{*}=0$ imply each other.

5. $m^{*}(M)$ is finite.

It was proved by $\mathrm{J}$. Radon $\dagger$ that the totality of these measures coincides with the totality of the measures representable by indefinite Lebesgue integrals,

$$
m^{*}(a)=\int_{a} f(P) d m
$$

where the point function $f(P)$ is positive on $M$ apart from a set of zero measure, and summable over $M$.

Now let $T$ be a one-to-one transformation of $M$ into itself, which transforms, as well as $T_{-1}$, measurable sets into measurable sets and sets of zero measure into sets of zero measure. The question to be investigated in this paper is the following: what intrinsic properties of $T$ involve the existence of a measure $m^{*}$ invariant under $T$ ?

Let us denote by $a_{\nu}$ the successive images of a point set $a=a_{0}$ obtained by successive application of $T$ or $T_{-1}$,

$\dagger \mathrm{J}$. Radon, Theorie und Anwendungen der absolut additiven Mengenfunktionen, Wiener Sitzungsberichte, vol. 122 (1913), p. 1299. 


$$
a_{\nu}=T_{\nu}(a) \quad(\nu=0, \pm 1, \pm 2, \cdots) .
$$

A set is called invariant (under $T$ ) if $a_{1}=a$. In the case of an arbitrary point set $b$, let us denote by $\{b\}$ the smallest invariant point set containing $b$. Obviously we have

$$
\{b\}=\sum_{-\infty}^{+\infty} b_{v} .
$$

The following rules are equally obvious:

$$
\begin{aligned}
(a+b+c+\cdots)_{\nu} & =a_{\nu}+b_{\nu}+c_{\nu}+\cdots, \\
(a b)_{\nu} & =a_{\nu} b_{\nu} \\
\{a+b+c+\cdots\} & =\{a\}+\{b\}+\{c\}+\cdots .
\end{aligned}
$$

Now we take a fixed point set $b$ and divide the invariant set $\{b\}$ into finitely or denumerably many different parts,

$$
\{b\}=c^{0}+c^{1}+c^{2}+\cdots,
$$

in such a way that $b$ contains at least one image of each of these parts,

$$
c^{(\nu)}=c_{n_{\nu}}^{\nu} \subset b \quad(\nu=0,1,2, \cdots) .
$$

Such a subdivision is always possible. For instance, we may set

$$
\begin{aligned}
& c^{0}=b_{0}=b, \\
& c^{1}=\left(b_{0}+b_{1}\right)-b_{0}, \\
& c^{2}=\left(b_{0}+b_{1}+b_{-1}\right)-\left(b_{0}+b_{1}\right), \\
& c^{3}=\left(b_{0}+b_{1}+b_{-1}+b_{2}\right)-\left(b_{0}+b_{1}+b_{-1}\right),
\end{aligned}
$$

In this case $b$ contains the images

$$
c^{(0)}=c_{0}^{0}, \quad c^{(1)}=c_{-1}^{1}, \quad c^{(2)}=c_{1}^{2}, \quad c^{(3)}=c_{-2}^{3}, \cdots .
$$

Instead of throwing $\{b\}$ into $b$ by means of subdivision and transformation of the parts we may throw any measurable subset $a$ of $\{b\}$ into $b$,

$$
\begin{aligned}
a \subset\{b\}, a & =c^{0}+c^{1}+c^{2}+\cdots ; c^{\nu} c^{\mu}=0, \nu & \neq \mu, \\
c^{(\nu)} & =c_{n_{\nu}} \subset b & (\nu=0,1,2, \cdots) .
\end{aligned}
$$

We set

$$
\sum_{b}^{a}=\sum_{v} m\left(c^{(v)}\right)
$$


and

$$
\mu(a)_{b}=\text { lower bound } \sum_{b}^{a}
$$

for all possible ways of throwing $a$ into $b$ according to (3) and ( $\left.3^{\prime}\right) . \mu$ is defined for all subsets $a$ of $\{b\}$ and nowhere negative. We may call $\mu(a)_{b}$ the "compressibility measure" of $a$ with respect to $b$. $†$ The compressibility measure has two important properties. It is absolutely additive and invariant under $T$, that is,

$$
\mu\left(a+a^{\prime}+a^{\prime \prime}+\cdots\right)_{b}=\mu(a)_{b}+\mu\left(a^{\prime}\right)_{b}+\mu\left(a^{\prime \prime}\right)_{b}+\cdots,
$$

$a, a^{\prime}, a^{\prime \prime}, \cdots$ being any denumerable set of point sets excluding each other. and

$$
\mu\left(a_{1}\right)_{b}=\mu(a)_{b} .
$$

The inequality

$$
\mu\left(a+a^{\prime}+a^{\prime \prime}+\cdots\right)_{b} \leqq \mu(a)_{b}+\mu\left(a^{\prime}\right)_{b}+\mu\left(a^{\prime \prime}\right)_{b}+\cdots
$$

for any sequence of sets is a well known consequence of the absolute additivity.

First we prove (7). From (3) and $\left(3^{\prime}\right)$ we have

$$
\begin{aligned}
& a_{1}=c_{1}^{0}+c_{1}^{1}+c_{1}^{2}+\cdots, \\
& \left(c_{1}^{p}\right)_{n_{\nu}-1}=c^{(\nu)} \subset b \quad(\nu=0,1,2, \cdots) \text {. }
\end{aligned}
$$

Thus each sum $\sum_{b}^{a}$ is a sum $\sum_{b}^{a_{1}}$. Conversely, by an analogous consideration, each sum $\sum_{b}^{a_{1}}$ is a sum $\sum_{b}^{a}$. Therefore their lower bounds coincide.

In order to prove (6) we set

$$
A=a+a^{\prime}+a^{\prime \prime}+\cdots, \quad A \subset\{b\},
$$

and throw $a$ into $b, a^{\prime}$ into $b, a^{\prime \prime}$ into $b$ and so on. These processes may be obviously combined into a single process of throwing the whole of $A$ into $b$. Thus each sum of sums $\sum_{b}^{a}+\sum_{b}^{a^{\prime}}+\sum_{b}^{a^{\prime \prime}}+\cdots$ represents a sum $\sum_{b}^{A}$. Conversely, let us throw $A$ into $b$,

$$
A=C^{0}+C^{1}+C^{2}+\cdots ; C^{\nu} C^{\mu}=0, \nu \neq \mu,
$$

$$
C^{(v)} \subset b \quad(\nu=0,1,2, \cdots),
$$

$$
\sum_{b}^{A}=\sum_{\nu} m\left(C^{(v)}\right)
$$

† Similarly we could define a "measure of expansion" by taking the upper bound instead of the lower bound of our sums. Probably our considerations can be simplified by using the measure of expansion. 
$C^{(v)}$ being an image of $C^{\nu}$ under a power $n_{\nu}$ of $T$. We may represent this process as a sequence of processes, by setting

$$
\left(a C^{\nu}\right)_{n_{\nu}}=c^{0 \nu},\left(a^{\prime} C^{\nu}\right)_{n_{\nu}}=c^{1 \nu}, \cdots \quad(\nu=0,1,2, \cdots),
$$

and

$$
\begin{aligned}
& a=\sum_{\nu} a C^{\nu}, c^{0 \nu} \subset b \quad(\nu=0,1,2, \cdots), \\
& a^{\prime}=\sum_{\nu} a^{\prime} C^{\nu}, c^{1 \nu} \subset b \quad(\nu=0,1,2, \cdots), \\
& \sum_{b}^{a}=\sum_{\nu} m\left(c^{0 \nu}\right), \quad \sum_{b}^{a^{\prime}}=\sum_{\nu} m\left(c^{1 \nu}\right), \cdots
\end{aligned}
$$

According to $C^{\nu} \subset A$ we obtain

$$
m\left(c^{0 v}\right)+m\left(c^{1 v}\right)+m\left(c^{2 v}\right)+\cdots=m\left(C^{(\nu)}\right),
$$

thus by (8)

$$
\sum_{b}^{A}=\sum_{b}^{a}+\sum_{b}^{a^{\prime}}+\sum_{b}^{a^{\prime \prime}}+\cdots
$$

Therefore the totality of the sums $\sum_{b}^{A}$ coincides with the totality of the sums of sums $\sum_{b}^{a}+\sum_{b}^{a^{\prime}}+\sum_{b}^{a^{\prime \prime}}+\cdots$, whence (6) immediately follows.

A further simple property of the compressibility measure is

$$
\mu(a)_{b^{\prime}} \leqq \mu(a)_{b}, b \subset b^{\prime}, a \subset\{b\} .
$$

Indeed, the totality of the sums $\sum_{b^{\prime}}^{a}$ contains the totality of the sums $\sum_{b}^{a}$. Let us put

$$
\lambda(b)=\mu(\{b\})_{b}
$$

For later purposes the following inequality may be derived:

$$
\lambda\left(\sum b^{\nu}\right) \leqq \sum \lambda\left(b^{\nu}\right)
$$

$b^{1}, b^{2}, \cdots$ being any sequence of point sets. Indeed, setting $b=\sum b^{\text {v }}$, we get by (2)

$$
\lambda(b)=\mu(\{b\})_{b}=\mu\left(\sum\left\{b^{\nu}\right\}\right)_{b}
$$

and by $\left(6^{\prime}\right)$ and (9)

$$
\mu\left(\sum\left\{b^{\nu}\right\}\right)_{b} \leqq \sum \mu\left(\left\{b^{\nu}\right\}\right)_{b} \leqq \sum \mu\left(\left\{b^{\nu}\right\}\right)_{b^{\nu}}=\sum \lambda\left(b^{\nu}\right) .
$$

Now we prove the following theorem: 
THEOREM 1. A necessary and sufficient condition for the existence of a finite and invariant measure $m^{*}$ over $M$ is that each invariant point set of positive measure contain $a$ set $b$ with

$$
0<\lambda(b)<\infty \text {. }
$$

The condition is necessary. Let $m^{*}$ be a finite invariant measure over $M$ given by (1) and let $A$ be any invariant point set of positive measure. Furthermore let $M^{n}$ be the (measurable) set of all points, for which

$$
1 / n<f(P)<n \text {. }
$$

Now the set of points with $f=0$ and $f=\infty$ has the measure zero, so that

$$
m\left(M^{n}\right) \rightarrow m(M), m\left(A M^{n}\right) \rightarrow m(A),
$$

$n$ tending to infinity. Hence $m\left(A M^{n}\right)>0$ for a suitable integer $n$. On setting $b=A M^{n}$ we conclude

$$
1 / n<m^{*}(c) / m(c)<n, . c \subset b, m(c)>0 .
$$

Throwing $\{b\}$ into $b$ we obtain by (12)

$$
\frac{1}{n} \sum m^{*}\left(c^{(\nu)}\right)<\sum m\left(c^{(\nu)}\right)=\sum_{b}^{\{t\}}<n \sum m^{*}\left(c^{(\nu)}\right) .
$$

On the other hand we have, since $c^{(\nu)}$ is an image of $c^{\nu}$ under a power of $T$,

$$
\sum m^{*}\left(c^{(\nu)}\right)=\sum m^{*}\left(c^{\nu}\right)=m^{*}\left(\sum c^{\nu}\right)=m^{*}(\{b\}) .
$$

Hence, according to (10),

$$
0<\frac{1}{n} m^{*}(\{b\}) \leqq \lambda(b)<n m^{*}(\{b\})<\infty .
$$

The condition is sufficient. A given invariant set of positive measure is supposed to contain a set $b$ with

$$
0<\lambda(b)<\infty .
$$

Let us designate a point set $a$ as a null set, if $\mu(a)_{b}=0$, and let us denote by $m^{\prime}$ the upper bound of the measures of all null sets. Furthermore let $a^{1}, a^{2}, \ldots$ be a sequence of null sets with $m\left(a^{\nu}\right) \rightarrow m^{\prime}, \nu \rightarrow \infty$. Now the invariant point set

$$
a^{\prime}=\sum_{\nu}\left\{a^{\nu}\right\}=\sum_{\nu, i} a_{i}^{\nu}
$$

is again a null set according to the properties of the compressibility measure, for we have 


$$
\mu\left(a^{\prime}\right)_{b}=\mu\left(\sum a_{i}^{p}\right)_{b} \leqq \sum \mu\left(a_{i}^{p}\right)_{b}=0 .
$$

According to the definition of the number $m^{\prime}$ we have $m\left(a^{\prime}\right)=m^{\prime}$. Furthermore we have $m\left(\{b\}-a^{\prime}\right)>0$, for $\{b\}$ cannot be a null set because of (10) and (13). Now $a^{\prime}$ is the largest null set, for according to the definition of $m^{\prime}$ the set $\{b\}-a^{\prime}$ cannot contain a further null set of positive measure. Thus

$$
\mu(a)_{b}, a \subset\{b\}-a^{\prime},
$$

defines a finite invariant measure $m^{*}$ over the invariant set $\{b\}-a^{\prime}$. We have also

$$
\mu\left(\{b\}-a^{\prime}\right)_{b}=\lambda(b) .
$$

We have to continue the formation of an invariant measure over larger and larger point sets, finally over the whole of $M$. For this purpose we denote by $\bar{m}$ the upper bound of the measures $m$ of all invariant point sets, over which a finite invariant measure $m^{*}$ can be defined. Let $M^{(1)}, M^{(2)}, \cdots$ be a corresponding sequence of invariant sets with

$$
\lim m\left(M^{(\nu)}\right)=\bar{m} .
$$

The finite invariant measure already found on $M^{(v)}$ may be denoted by $m_{v}^{*}$. Then we are able to define an invariant measure $m^{*}$ over the whole of

by setting

$$
M^{\prime}=\sum_{1}^{\infty} M^{(v)}
$$

$$
\begin{aligned}
& m^{*}=\alpha_{1} m_{1}^{*} \text { over } M^{(1)}, \\
& m^{*}=\alpha_{n} m_{n}^{*} \text { over } \sum_{1}^{n} M^{(v)}-\sum_{1}^{n-1} M^{(v)}, n>1,
\end{aligned}
$$

$\alpha_{1}, \alpha_{2}, \cdots$ being a sequence of positive numbers. Clearly the total invariant measure

$$
m^{*}\left(M^{\prime}\right)=m^{*}\left(M^{(1)}\right)+\sum_{n=2}^{\infty} m^{*}\left[\sum_{1}^{n} M^{(v)}-\sum_{1}^{n-1} M^{(v)}\right]
$$

becomes finite by suitable choice of the numbers $\alpha_{v}$. Thus a finite invariant $m^{*}$ may be defined over the whole of the invariant set $M^{\prime}$. Now the invariant point set $M-M^{\prime}$ must have the measure zero, because otherwise we could continue the formation of an invariant $m^{*}$ over $M-M^{\prime}$. Hence the finite invariant $m^{*}$ constructed above applies to the whole of $M$.

Simpler conditions may be obtained for particular types of invariant measures. Let us call a measure $m^{*}$ a measure of the order of $m$, if 


$$
m^{*}(a) / m(a)
$$

lies between positive bounds for all sets $a \subset M$ of positive measure. The following theorem was proved by Birkhoff and Smith: $\dagger$

Theorem 2. A necessary and sufficient condition for the existence of an invariant measure $m^{*}$ of the order of $m$ is that

$$
m\left(a_{v}\right) / m(a) \geqq \text { const. }>0 ; \nu=0, \pm 1, \pm 2, \cdots,
$$

the constant being independent not only of $\nu$ but also of the set a.

The condition is necessary, for

$$
1 / x \leqq m^{*}(a) / m(a) \leqq x, a \subset M,
$$

implies

$$
1 / x^{2}=m^{*}\left(a_{v}\right) / x^{2} m^{*}(a) \leqq m\left(a_{v}\right) / m(a) \leqq x^{2} m^{*}\left(a_{v}\right) / m^{*}(a)=x^{2} .
$$

The condition is sufficient. We choose

$$
m^{*}(a)=\mu(a)_{M} \text {. }
$$

If the inequalities

$$
1 / y \leqq m\left(a_{v}\right) / m(a) \leqq y
$$

are satisfied for any set $a$ and any number $\nu$, we obtain, by a subdivision of $a$,

$$
\begin{gathered}
a=\sum c^{\nu} ; c^{\nu} c^{\mu}=0, \nu \neq \mu, \\
\sum m\left(c^{(\nu)}\right) \leqq y \sum m\left(c^{\nu}\right)=y m(a),
\end{gathered}
$$

$c^{(\nu)}$ being an image of $c^{\nu}$ under a power of $T$. Similarly we get

$$
\sum m\left(c^{(\nu)}\right) \geqq \sum m\left(c^{\nu}\right) / y=m(a) / y,
$$

whence

$$
1 / y \leqq \mu(a)_{M} / m(a) \leqq y,
$$

for any set $a \subset M$ of positive measure.

3. Further preliminary considerations. If no finite and invariant measure $m^{*}$ exists over $M$, we infer from Theorem 1 the existence of an invariant point set $A, m(A)>0$, such that the symbol $\lambda(b)$ does not take values except

$$
\lambda(b)=0, \lambda(b)=\infty \text {, }
$$

$b$ being any subset of $A$. This behavior of $\lambda$ in the case of non-existence of a finite invariant $m^{*}$ may be illustrated by a simple example. paper.

$\dagger$ G. D. Birkhoff and P. Smith, loc. cit., §4. The special sums $\sum_{M}^{a}$ were first introduced in this 
Let $M$ be the linear interval $0<x<1$ and let $m$ be the ordinary measure on $M$ in the sense of Lebesgue. Let $T$ be a transformation which leaves $x=0,1$ invariant and shifts all inner points to the left. The images $x$, of a point $x=x_{0}$ clearly satisfy the conditions

$$
x_{\nu}>x_{v+1}, \lim _{\nu=+\infty} x_{\nu}=0, \lim _{\nu=-\infty} x_{\nu}=1 ;
$$

in other words, the intervals

$$
I_{\nu}: x_{\nu}>x \geqq x_{\nu+1}
$$

exclude each other and fill up the whole of $M$, also $\left\{I_{0}\right\}=M$. A finite invariant $m^{*}$ cannot exist, for $m^{*}\left(I_{v}\right)$ would be independent of $\nu$, and $m^{*}(M)$ $=\sum m^{*}\left(I_{v}\right)$. It can be shown that $\lambda=\infty$ holds for each interval the end points of which are inner points of $M$. On the other hand $\lambda=0$ holds for any interval having an end point at 0 or 1 . Consider for instance the two intervals $I_{0}$ and $I^{\prime}=\sum_{0}^{\infty} I_{v}$. We may throw $M=\left\{I_{0}\right\}$ into $I_{0}$ by

$$
M=\sum_{-\infty}^{+\infty} I_{\nu},\left(I_{\nu}\right)_{-}=I_{0}
$$

The corresponding sum $\sum_{I_{0}}^{M}$ is infinite, and it is readily seen that this sum is not altered by throwing $M$ into $I_{0}$ differently. Hence $\lambda\left(I_{0}\right)=\infty$. On the other hand let us throw $M$ into the interval $I^{\prime}=\sum_{0}^{\infty} I_{v}$ by

$$
M=\sum_{-\infty}^{+\infty} I_{\nu},\left(I_{\nu}\right)_{n} \subset I^{\prime}, \nu \geqq 0 ; \quad\left(I_{\nu}\right)_{n-2 v-1} \subset I^{\prime}, \nu<0,
$$

$n$ being a positive integer. In this case we have

$$
\sum_{I^{\prime}}^{M}=\sum_{0}^{\infty} m\left(I_{n+v}\right)+\sum_{-1}^{-\infty} m\left(I_{n-v-1}\right)=2 m\left(\sum_{n}^{\infty} I_{\nu}\right)
$$

which can be made arbitrarily small by a suitable choice of $n$. Hence $\lambda\left(I^{\prime}\right)=0$. It may be remarked that an infinite measure, invariant under $T$, may be constructed. $m^{*}$ may be arbitrarily chosen on $I_{0}$ and automatically continued on the images $I$, by $T$.

The fact that $\lambda=\infty$ holds for each closed interval $\subset M$ in our example is a particular case of a general fact concerning the values of $\lambda$, if no finite invariant $m^{*}$ exists. We prove

THEOREM 3. There are only the following two possibilities, in case no finite invariant $m^{*}$ exists: 
( $\alpha$ ) an invariant point set $A$ of positive measure exists such that $\lambda(b)=0$ for any $b \subset A$;

( $\beta$ ) an invariant point set $A$ of positive measure and an infinite sequence of increasing sets filling $A$,

$$
A^{1} \subset A^{2} \subset A^{3} \subset \cdots, \sum A^{v}=A,
$$

exist such that $\lambda(b)=\infty$, whenever $m(b)>0$ and $b \subset A^{\nu}$ for a sufficiently large $\nu$.

We observe that $(\alpha)$ and $(\beta)$ do not necessarily exclude each other (in this case the $A$ of $(\alpha)$ is of course different from the $A$ of $(\beta))$. We know already the existence of an invariant set $A$ of positive measure such that either $\lambda(b)=0$ or $\lambda(b)=\infty$ for any $b \subset A$. Excluding $(\alpha)$ we assume $\lambda(b)=\infty$ for a suitable $b \subset A$. Any set $a \subset b$ satisfies either the equation $\lambda(a)=0$ or $\lambda(a)=\infty$. Let us denote by $\bar{m}$ the upper bound of the measures of all sets $a \subset b$ with $\lambda(a)=0$, and let us select a sequence of such sets contained in $b$,

$$
b^{1}, b^{2}, \cdots, \lambda\left(b^{\nu}\right)=0, \quad \lim m\left(b^{\nu}\right)=\bar{m} .
$$

According to (11) the point set

$$
b^{\prime}=\sum_{1}^{\infty} b^{v}
$$

satisfies again the equation $\lambda\left(b^{\prime}\right)=0$, and we conclude $m\left(b^{\prime}\right)=\bar{m}$ according to the definition of the upper bound $\bar{m}$. The set $b-b^{\prime}$ has a positive measure, for otherwise we would have $\lambda(b)=\lambda\left(b^{\prime}\right)=0$ in contradiction to our assumption $\lambda(b)=\infty$. Now, $b^{\prime}$ is the largest set with $\lambda\left(b^{\prime}\right)=0$ according to the definition of $\bar{m}$ and according to (11), and each subset $a$ of $b-b^{\prime}, m(a)>0$, satisfies $\lambda(a)=\infty$.

We have hereby constructed a point set $c$ of positive measure such that $\lambda(a)=\infty$ holds for any subset $a$ of $c, m(a)>0$.

In order to construct a sequence of sets indicated in the condition $(\beta)$ of Theorem 3, we may start with the following preliminary considerations. According to the properties of the transformation $T$ the point set function $m\left(a_{v}\right)$, regarded as a function of the set $a, \nu$ being a given integer, has precisely all properties of a measure $m^{*}(a)$. Thus we may represent it as an indefinite integral in the sense of Radon,

$$
m\left(a_{\nu}\right)=\int_{a} \phi_{\nu}(P) d m
$$

$\phi$, being positive almost everywhere and summable over $M$. Under well known restrictions for $M$ and $T, \phi_{\nu}$ is the Jacobian of $T_{\nu}$. For a given integer 
$\nu$ a positive number $\epsilon_{\nu}$ can always be found so that the measure of the set of all points $P$ with $\phi_{\nu}(P) \leqq \epsilon_{\nu}$ is less than $m(c) 2^{-\nu-1}, c$ being the set constructed above. The sum of these point sets $(\nu= \pm 1, \pm 2, \cdots)$ has a measure less than

$$
m(c) \sum_{1}^{\infty} 2^{-\nu-1}=m(c) / 2 .
$$

Thus the complementary set $e$ of this sum with respect to the set $c$ has a measure $m(e)>m(c) / 2>0$. The set $e$ is precisely the set of all points $P$ of $c$, for which the inequalities $\phi_{\nu}(P)>\epsilon_{\nu}$ hold simultaneously, $\nu= \pm 1, \pm 2, \cdots$. Now we are able to prove that the point sets

$$
A=\{e\} ; A^{1}, A^{2}, A^{3}, \cdots,
$$

where

$$
A^{2 n}=\sum_{1-n}^{n} e_{\nu}, n>0 ; A^{2 n+1}=\sum_{-n}^{n} e_{\nu}, n \geqq 0,
$$

satisfy the condition $(\beta)$ of Theorem 3. For this purpose we write

$$
E^{1}=A^{1}=e, E^{\nu+1}=A^{\nu+1}-A^{\nu}, \nu>0 .
$$

Any two of the sets $E^{v}$ have no points in common, and it is evident that

(16) and (17) imply

$$
\sum_{1}^{\infty} E^{\nu}=A
$$

$$
E_{h}^{k+1} \subset e,
$$

where $h=-(k+1) / 2$ for $k$ odd, $h=k / 2$ for $k$ even. According to (14) and to the definition of the point set $e$ the inequalities

$$
m\left(a_{-h}\right) \geqq \epsilon_{-h} m(a), a \subset e \quad\left(\epsilon_{-h}>0\right),
$$

are satisfied by any subset $a$ of $e$.

Now we know already that $\lambda=\infty$ holds for any subset $a$ of $A^{1}=e$ with positive measure. We may then prove $(\beta)$ by complete induction, assuming that $\lambda(a)=\infty$ holds for any subset $a \subset A^{k}, m(a)>0$.

We throw $A$ into $A^{k+1}$,

$$
A=\sum_{\nu} c^{\nu}, c^{(v)} \subset A^{k+1},
$$

where $c^{(v)}$ is the image of $c^{\nu}$ under the $n_{\nu}$ th power of $T$, and we set

$$
\sum^{k+1}=\sum_{v} m\left(c^{(v)}\right) \text {. }
$$


According to (17) we have

$$
A^{k+1}=A^{k}+E^{k+1}, A^{k} E^{k+1}=0 .
$$

We subdivide the parts $c^{\prime}$ of $A$ by setting

$$
c^{\nu}=a^{\nu}+b^{\nu},
$$

where $a^{v}, b^{v}$ are defined by the equations

$$
a^{(v)}=c^{(\nu)} A^{k}, b^{(v)}=c^{(\nu)} E^{k+1},
$$

$a^{(v)}, b^{(v)}$ being the images of $a^{\nu}, b^{\nu}$ under the $n_{\nu}$ th power of $T$, respectively. By (21) we get

$$
\sum^{k+1}=\sum_{\nu} m\left(a^{(\nu)}\right)+\sum_{\nu} m\left(b^{(\nu)}\right) .
$$

From $\left(23^{\prime}\right)$,

$$
a^{(v)} \subset A^{k}
$$

on the other hand, from (18) and $\left(23^{\prime}\right)$,

$$
b_{h}^{(v)} \subset e=A^{1} \subset A^{k} .
$$

Thus $A=\sum a^{\nu}+\sum b^{\nu}$ together with (25) and (25') represents a way of throwing $A$ into $A^{k}$. Since, by hypothesis, $\lambda\left(A^{k}\right)=\infty$, we have

$$
\sum^{k}=\sum_{\nu} m\left(a^{(\nu)}\right)+\sum_{\nu} m\left(b_{h}^{(\nu)}\right)=\infty .
$$

Applying (19) to $a=b_{h}^{(\nu)}\left(\right.$ see $\left.\left(25^{\prime}\right)\right)$ we obtain

$$
\sum_{\nu} m\left(b^{(p)}\right) \geqq \epsilon_{-h} \sum_{\nu} m\left(b_{h}^{(p)}\right) ;
$$

thus, by comparison with (24) and (26),

$$
\sum^{k+1} \geqq \gamma \sum^{k}, \gamma=\min \left(1, \epsilon_{-h}\right),
$$

and $\sum^{k+1}=\infty, \lambda\left(A^{k+1}\right)=\infty$. An analogous consideration yields $\lambda(a)=\infty$ for any $a \subset A^{k+1}, m(a)>0$, which completes the proof of Theorem 3 .

4. Images by division and invariant measures. Concerning the notion of the image by division we shall only require that the different parts exclude each other in the sense of measure theory, i.e., that they have at most sets of zero measure in common. However, the greater generality of this notion is readily seen to be apparent. Let us call a set $A$ a "proper part" of a set $B$, if $A \subset B$ and $m(A)<m(B)$. We note the following simple results. 
LEMMA 1. If each image by division of the whole of $M$ has the measure $m(M)$, no set $A$ possesses an image by division forming a proper part of $A$.

This simply means, that the intrinsic incompressibility of the whole of $M$ implies the incompressibility of the subsets. Let $A^{\prime}$ be an image by division of $A$, and let $A^{\prime} \subset A, m\left(A^{\prime}\right)<m(A)$. We put

$$
M=(M-A)+A, M^{\prime}=(M-A)+A^{\prime} .
$$

Obviously $M^{\prime}$ is an image by division of $M$ with

$$
m\left(M^{\prime}\right)=m(M-A)+m\left(A^{\prime}\right)<m(M-A)+m(A)=m(M) .
$$

LEMмa 2. The compressibility measure of a set $A$ with respect to an image by division $A^{\prime}$ of $A$ is always finite.

The corresponding divisions of $A$ and of $A^{\prime}$,

$$
A=\sum a^{\nu}, A^{\prime}=\sum a^{(\nu)}
$$

yield a particular sum

Hence

$$
\sum_{A^{\prime}}^{A}=\sum m\left(a^{(v)}\right)=m\left(A^{\prime}\right)
$$

$$
\mu(A)_{A^{\prime}} \leqq m\left(A^{\prime}\right) .
$$

The following lemma will be useful for later discussions:

LemMA 3. Let $B^{\prime}$ be an image by division of $B$ and let $A \supset B^{\prime}$. If

$$
m\left(A-B^{\prime}\right)>0,\left\{A-B^{\prime}\right\} \subset B,
$$

the second inequality holding apart from a set of zero measure, then $M$ possesses an image by division that is a proper part of $M$.

Let

$$
B=\sum b^{\nu}, \quad B^{\prime}=\sum b^{(\nu)} ; \quad b^{\nu} b^{\mu}=b^{(\nu)} b^{(\mu)}=0, \quad \nu \neq \mu ; \quad b^{(\nu)}=b_{n_{\nu}}^{\nu}
$$

We set

$$
E=\left\{A-B^{\prime}\right\}, E^{\prime}=B^{\prime}\left\{A-B^{\prime}\right\} .
$$

Now $E^{\prime}$ is an image by division of $E$, for (27), (28) and (29) yield

$$
E=B E=\sum b^{\nu} E ; E^{\prime}=B^{\prime} E=\sum b^{(\nu)} E=\sum\left(b^{\nu} E\right)_{n_{\nu}},
$$

the first of these equations holding apart from a set of zero measure. From (29) we conclude that

$$
E^{\prime} \subset E, E-E^{\prime} \supset\left(A-B^{\prime}\right)\left\{A-B^{\prime}\right\} \supset A-B^{\prime} .
$$


Hence according to $(27), m\left(E-E^{\prime}\right)>0$, i.e. $E^{\prime}$ is a proper part of $E$. Lemma 1 completes the proof.

We need two further simple results.

LEMMA 4. If $m(a)>0, m(b)>0, \mu(a)_{b}=0$, $a$ point set $a^{\prime}$ and an integer $n$ can always be found to satisfy the conditions

$$
\begin{gathered}
a^{\prime} \subset a, a_{n}^{\prime} \subset b, \\
0<m\left(a_{n}^{\prime}\right)<m\left(a^{\prime}\right) / 2 .
\end{gathered}
$$

We can always throw $a$ into $b$ in such a way that

$$
\sum m\left(c^{(\nu)}\right)<\frac{1}{2} m(a)=\frac{1}{2} \sum m\left(c^{\nu}\right) ; a=\sum c^{\nu}, c^{(v)}=c_{n,}^{\nu} c b .
$$

Thus the inequality $m\left(c^{(v)}\right)<m\left(c^{\nu}\right) / 2$ must hold for some $\nu . a^{\prime}=c^{\nu}, n=n$, satisfy (30).

Lemma $4^{\prime}$. If $m(a)>0, m(b)>0, \mu(a)_{b}=\infty$, a set $a^{\prime}$ and an integer $n$ can always be found to satisfy the conditions

$$
\begin{gathered}
a_{n}^{\prime} \subset a, a^{\prime} \subset b, \\
0<m\left(a_{n}^{\prime}\right)<m\left(a^{\prime}\right) / 2 .
\end{gathered}
$$

By any way of throwing $a$ into $b$ we get

$$
\sum m\left(c^{(v)}\right)=\infty ; a=\sum c^{\nu}, c^{(\nu)}=c_{n_{\nu}}^{\nu} \subset b .
$$

The inequality $m\left(c^{(v)}\right)>2 m\left(c^{\nu}\right)$ must therefore hold for some $\nu$. Then, $a^{\prime}=c^{(v)}$, $n=-n$, satisfy (31).

We are now prepared to prove the main

ThEOREM 4. A necessary and sufficient condition for the existence of a finite invariant measure $m^{*}$ over the whole of $M$ is that $m\left(M-M^{\prime}\right)=0$ holds for each image by division $M^{\prime}$ of $M$.

It remains to prove that the condition is sufficient. Under the assumption that no finite invariant $m^{*}$ exists over $M$, we shall construct an image by division of $M$ forming a proper part of $M$. With regard to Theorem 3, we have to treat separately the two cases $(\alpha)$ and $(\beta)$.

( $\alpha$ ) An invariant set $A$ exists such that $\lambda(b)=0$ holds for any subset $b$ of $A$. From $\mu(a)_{b} \leqq \mu(\{b\})_{b}=\lambda(b)$ we conclude that

$$
\mu(a)_{b}=0
$$

holds for any set $b \subset A$ and any set $a \subset\{b\}$.

We may arrange point sets in certain groups $G_{0}, G_{1}, G_{2}, \ldots$ A set $a$ be- 
longs to $G_{n}$, if $2^{-n} m(A)>m(a) \geqq 2^{-n-1} m(A)$. The group $G_{n}$ cannot contain more than $2^{n+1}$ sets belonging to a sequence of sets which exclude each other. According to Lemma 4 (with $a=b=A$ ) we can find a set $a^{1} \subset A$ and an image $a^{(1)}$ under a suitable power of $T$ such that

$$
m\left(a^{(1)}\right)<m\left(a^{1}\right) / 2 .
$$

$a^{1}$ belongs to a group $G_{\alpha}$. We choose $a^{1}, a^{(1)}$ so that $\alpha$ is as small as possible. Now we select, if possible, a second set $a^{2}$ and an image $a^{(2)}$ under a suitable power of $T$ which satisfy the conditions

$$
\begin{gathered}
a^{2} \subset A, a^{1} a^{2}=a^{(1)} a^{(2)}=0, \\
m\left(a^{(2)}\right)<m\left(a^{2}\right) / 2 .
\end{gathered}
$$

We require again the index $\beta$ of the group $G_{\beta}$ to which $a^{2}$ belongs to be as small as possible, provided that (33) is satisfied. By continuing this process as far as possible we obtain a finite or infinite sequence of sets $a^{1}, a^{2}, \cdots$ and an associated set of images $a^{(1)}, a^{(2)}, \cdots$ satisfying the conditions

$$
\begin{gathered}
a^{\nu} \subset A, a^{\nu} a^{\mu}=a^{(\nu)} a^{(\mu)}=0, \nu \neq \mu, \\
m\left(a^{(\nu)}\right)<m\left(a^{\nu}\right) / 2,
\end{gathered}
$$

where in succession the index $\alpha_{n}$ of the group $G$, to which $a^{n}$ belongs, is chosen as small as possible. We have

$$
\alpha_{1} \leqq \alpha_{2} \leqq \alpha_{3} \leqq \cdots .
$$

If the sequence is infinite, we necessarily have $\alpha_{v} \rightarrow \infty$, whence follows that the process cannot be continued further. In any case, we assume the process to be continued as far as possible.

The point sets

$$
B=\sum a^{\prime}, B^{\prime}=\sum a^{(v)}
$$

are images by division of each other. From (34) we have

$$
m\left(B^{\prime}\right)<m(B) / 2<m(A) .
$$

On setting

$$
b=A-B^{\prime}, a=(A-B)\{b\},
$$

we therefore have

$$
m(b)>0 \text {. }
$$

Now we show that necessarily $m(a)=0$, the case $m(a)>0$ leading to a contradiction with our assumption that the process cannot be carried further. 
According to Lemma 4 we may indeed find a set $a^{s}$ and a suitable image $a^{(s)}$ so that

$$
a^{s} \subset a, a^{(s)} \subset b, m\left(a^{(s)}\right)<m\left(a^{s}\right) / 2 .
$$

According to (35) the first and the second inequality are obviously equivalent to

$$
a^{s} \subset A, a^{s} a^{\nu}=a^{(s)} a^{(\nu)}=0,1 \leqq \nu<s,
$$

so that (34) is satisfied for the former sets $a^{\nu}$ together with $a^{s}$. Thus we must have $m(a)=0$, i.e., $\left\{A-B^{\prime}\right\} \subset B$ holds apart from a set of measure zero. In this case Lemma 3 finishes the proof. In conclusion, the process can either be continued till the sets $a^{\nu}$ fill up the whole of $A$, or it stops before. The latter case is by no means unfavorable, as Lemma 3 automatically provides for it.

$(\beta)$ An invariant set $A, m(A)>0$, exists having the following property. For any given number $\epsilon>0$ a set $\bar{A} \subset A$ can be found such that

$$
m(A-\bar{A})<\epsilon
$$

while for any $b \subset \bar{A}, m(b)>0$, we have

$$
\lambda(b)=\infty \text {. }
$$

The treatment of this case follows much the same line as in the case $(\alpha)$, the only complication being due to the fact that the equation $\mu(a)_{b}=\infty$ is not satisfied by each $b \subset \bar{A}, a \subset\{b\}, m(a)>0$.

We start by fixing a set $\bar{A}$ such that $\left(36^{\prime}\right)$ holds for any $b \subset \bar{A}, m(b)>0$. According to Lemma $4^{\prime}$ with $b=\bar{A}, a=\{b\}, \mu(a)_{b}=\lambda(\bar{A})=\infty$, we may select a first set $a^{1}$ and a suitable image $a^{(1)}$ so that

$$
\begin{gathered}
a^{1} \subset \bar{A}, \\
m\left(a^{(1)}\right)<m\left(a^{1}\right) / 2 .
\end{gathered}
$$

We proceed exactly as in the case $(\alpha)$ by constructing a sequence of sets $a^{\nu}$ and of associated images which satisfy the conditions

$$
\begin{gathered}
a^{\nu} \subset \bar{A}, a^{\nu} a^{\mu}=a^{(\nu)} a^{(\mu)}=0, \nu \neq \mu, \\
m\left(a^{(\nu)}\right)<m\left(a^{\nu}\right) / 2 .
\end{gathered}
$$

We assume again the process to be carried on as far as possible. We set now

$$
B=\sum a^{(\nu)}, B^{\prime}=\sum a^{\nu},
$$

these sets being images by division of each other, and

$$
b=\bar{A}-B^{\prime}, a=(A-B)\{b\}
$$


The assumption $m(a)>0$ is again in contradiction with the assumption that our process cannot be continued. In order to show this, we note first that

$$
\mu(a)_{b}=\infty,
$$

an equation which will be proved below. Supposing (38) to be proved we may apply Lemma 4 and accordingly find a set $a^{s}$ and an image $a^{(s)}$ such that

$$
\begin{gathered}
a^{s} \subset \bar{A}-B^{\prime}, a^{(s)} \subset a \subset A-B, \\
m\left(a^{(s)}\right)<m\left(a^{s}\right) / 2 .
\end{gathered}
$$

Thus a continuation would be possible. We conclude therefore that $m(a)=0$, i.e., that $\left\{\bar{A}-B^{\prime}\right\} \subset B$ holds apart from a point set of measure zero. If now $m(b)=m\left(\bar{A}-B^{\prime}\right)>0$, we may apply Lemma 3 , replacing the $A$ occurring there by our present $\bar{A}$. If, on the other hand, $m(b)=0$, the point set $\bar{A}$ is filled by the sets $a^{p}$. We may then continue our process by choosing a larger $\bar{A}$ and by filling, if possible, the remainder of this new $\bar{A}$ with respect to the former $\bar{A}$. Either the remainder can be filled up by new sets $a^{\nu}$, or the process stops before. The latter case is settled by Lemma 3. On setting $\bar{A}=A^{1}, A^{2}$, $A^{3}, \ldots$ (see Theorem 3 ) in succession we finally infer that $M$ possesses an image by division which is a proper part of $M$.

It remains to prove (38). For this purpose let us set

$$
C=B\{b\}, \quad C^{\prime}=B^{\prime}\{b\} .
$$

As $\{b\}$ is an invariant point set, $C$ and $C^{\prime}$ must be images by division of each other. (37) and (39) yield

$$
\{b\}=a+C, a C=0 .
$$

Since $b=\bar{A}-B^{\prime}$ and $C^{\prime} \subset B^{\prime}$, the set

$$
E=b+C^{\prime}
$$

must be part of $\bar{A}$. With regard to the definition of the set $\bar{A}$ we have

From (39) we obtain

$$
\lambda(E)=\mu(\{E\})_{E}=\infty .
$$

$$
\left\{C^{\prime}\right\} \subset\{b\},\left\{b+C^{\prime}\right\}=\{b\}+\left\{C^{\prime}\right\}=\{b\} ;
$$

thus according to (40) and (41),

$$
\mu(\{E\})_{E}=\mu(\{b\})_{E}=\mu(a)_{E}+\mu(C)_{E}=\infty .
$$

By (9) and (41) we finally obtain

$$
\mu(a)_{E} \leqq \mu(a)_{b}, \mu(C)_{E} \leqq \mu(C)_{C^{\prime}},
$$

where $\mu(C)_{C}$, is a finite number according to Lemma 2. Hence $\mu(a)_{b}=\infty$. 
5. Some remarks on images by finite division. It is an outstanding question, whether the weaker supposition, that no set goes into a proper part of itself under $T$, guarantees the existence of a finite invariant $m^{*}$. According to Theorem 4 this would be equivalent to the question whether a set going into a proper part of itself under $T$ can be found, if a suitable image by division of $M$ forms a proper part of $M$. It is rather doubtful whether the anwer to this question is in the affirmative. However, it may be remarked that the main difficulty lies in the use of infinitely many parts in the concept of the image by division, for in case of divisions into a finite number of parts our question is affirmed by

Theorem 5. If a point set $A$ possesses an image by finite division which is a proper part of $A$, a point set can always be constructed, which goes into a proper part of itself under $T$.

The proof of Lemma 1 shows that the whole of $M$ possesses an image by finite division $M^{\prime}$,

$$
\begin{gathered}
M=\sum_{1}^{k} a^{\nu}, M^{\prime}=\sum_{1}^{k} a^{(\nu)}, a^{(\nu)}=a_{n_{\nu}}^{\nu} \\
a^{\nu} a^{\mu}=a^{(\nu)} a^{(\mu)}=0, \nu \neq \mu,
\end{gathered}
$$

so that

$$
M^{\prime} \subset M, m\left(M-M^{\prime}\right)>0 .
$$

We may assume that

$$
n_{\nu}>1 \quad(\nu=1,2, \cdots, k),
$$

for otherwise we might use the set $M_{n}^{\prime}$ instead of $M^{\prime}, n$ being sufficiently large. Now let us consider the array, $k \geqq 1$,

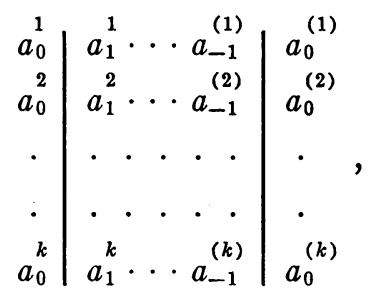

where the numbers of elements in the different rows are $n_{1}+1, n_{2}+1, \ldots$ successively. We denote by $E^{i}$ the set of all points contained in $i$ or more point sets standing between the two vertical lines, and we put $E^{0}=M$. These sets decrease, $i$ increasing,

$$
M=E^{0} \supset E^{1} \supset E^{2} \supset \cdots \supset E^{s}, E^{s+1}=0,
$$


where $s \leqq \sum_{1}^{k}\left(n^{\nu}-1\right)$. Now we set

$$
A^{i}=E^{i-1}, B^{i}=E^{i}+M^{\prime} E^{i-1} \quad(i=1,2, \cdots, s+1) .
$$

As $a_{\mu}^{p} \subset M$ and $\sum a^{\nu}=M$, each point of $E^{i-1}$ belongs to a suitable $a^{\nu}$. Thus each point of $E^{i-1}$ belongs to $i$ or more point sets on the left of the right vertical lines in (45). Conversely, each point with the latter property belongs to $i-1$ or more sets between the vertical lines, because it cannot belong to two different sets $a_{8}$ according to (42). $A^{i}$ is therefore the set of all points contained in $i$ or more'sets on the left of the right vertical line in (45).

It is equally obvious that each point of $B^{i}$ belongs to $i$ or more sets on the right of the left line in (45). Conversely, each point with the latter property either belongs to $E^{i}$ or it belongs to some $a_{0}^{(\nu)}$. But in this case it must be contained in $E^{i-1}$, because the sets $a_{0}^{(v)}$ exclude each other. Thus $B^{i}$ is exactly the set of all points contained in $i$ or more sets on the right of the left vertical line. By this consideration we clearly obtain

$$
B^{i}=A_{1}^{i} \quad(i=1,2, \cdots, s+1) .
$$

(46), (47) and (48) yield

$$
A_{1}^{i} \subset A^{i} .
$$

Now we prove that $A_{1}^{i}$ must be a proper part of $A^{i}$ for a suitable $i$. From (46), (47) and (48) we easily get

$$
A^{i}-A_{1}^{i}=\left(M-M^{\prime}\right)\left(E^{i-1}-E^{i}\right) \quad(i=1,2, \cdots, s+1),
$$

these point sets excluding each other. Hence follows

$$
\sum_{1}^{s+1} m\left(A^{\nu}-A_{1}^{\nu}\right)=m\left[\left(M-M^{\prime}\right)\left(E^{0}-E^{s+1}\right)\right]=m\left(M-M^{\prime}\right)>0
$$

according to (43), thus yielding $m\left(A^{\nu}-A_{1}^{\nu}\right)>0$ for some $\nu$.

Generalisations. So far we have considered only the case of a single transformation of $M$ into itself. Apart from $\$ 5$, however, all notions and results may easily be extended to very general groups of transformations. This may be briefly outlined in the case of a linear one-parameter group $T_{t}$, $-\infty<t<+\infty$,

$$
T_{t} T_{s}=T_{s+t},
$$

of transformations of $M$ into itself (steady flow on $M$ ). Every transformation of the group is supposed to have the properties stated in $\$ 2$. Concerning the dependence upon $t$ we suppose that 


$$
\lim _{t=0} m\left(A T_{t}(B)\right)=m(A B)
$$

holds for any two measurable sets $A, B$. This general supposition is certainly fulfilled if $M$ is an analytic manifold and if $T_{t}(P)$ is analytic in $P$ and $t$.

A measurable point set $A$ is called invariant under the group if, for every $t, T_{t}(A)$ coincides with $A$ apart from a point set of measure zero. The smallest invariant set $\{A\}$ containing a. set $A$ is defined by

$$
\{A\}=\sum_{r} T_{r}(A)
$$

where $r$ runs through the sequence of the rational numbers. $\{A\}$ is obviously invariant under all $T_{t}$ with a rational index; in other words, the equations

$$
m\left(\{A\} T_{t}\{A\}\right)=m(\{A\})=m\left(T_{t}\{A\}\right)
$$

hold for all rational values of $t$. From the above continuity supposition we infer that they hold for any $t$, i.e., that $\{A\}$ is invariant under the group. Notions such as the compressibility measure and the image by division admit of an obvious extension. As all our considerations remain the same, Theorem 4 indicates also in the case of our group the necessary and sufficient condition for the existence of a measure $m$, invariant under the group $T_{t}$.

HaRVARD University, Cambridge, Mass. 